\title{
Análisis de la actividad laboral de los profesionales sanitarios en formación de la Región de Murcia ante la pandemia por COVID- 19
}

\section{Analysis of the work activity of health professionals in training in the Region of Murcia in the face of the COVID-19 pandemic}

\author{
Domingo A. Sánchez ${ }^{1 *}$, Pablo Madrigal Laguía ${ }^{2}$, Santiago Serrano Porta ${ }^{3}$, José \\ Ángel Guardiola Olmos ${ }^{4}$ y Marta Cañadilla Ferreira ${ }^{5}$ \\ 1 Servicio de Oncología Médica, Hospital Clínico Universitario Virgen de la Arrixaca; Servicio \\ Murciano de Salud, domingoa.sanchez@carm.es, https://orcid.org/0000-0003-2073-0679 \\ 2 Unidad Docente Multiprofesional de Atención Familiar y Comunitaria Cartagena-Mar \\ Menor; Complejo Universitario Hospitalario de Cartagena; Servicio Murciano de Salud, \\ pablo.madrigal.laguia@gmail.com, https://orcid.org/0000-0003-1886-271X \\ 3 Unidad Docente Multiprofesional de Atención Familiar y Comunitaria Cartagena-Mar Menor; \\ Complejo Universitario Hospitalario de Cartagena; Servicio Murciano de Salud, \\ yuago@icloud.com, https://orcid.org/0000-0002-2174-2906 \\ 4 Unidad Docente de Pediatría y sus Áreas Específicas; Hospital Clínico Universitario Virgen \\ de la Arrixaca; Servicio Murciano de Salud, jangelguardiola@gmail.com, \\ https://orcid.org/0000-0002-0474-5902 \\ 5 Unidad Docente de Medicina Preventiva y Salud Pública de la Región de Murcia; Servicio \\ Murciano de Salud, martcafe89@gmail.com, https://orcid.org/0000-0001-7930-782X \\ * Correspondencia: domingoa.sanchez@carm.es
}

Recibido: 7 de abril de 2020; Aceptado: 3 de mayo de 2020; Publicado: 4 de mayo de 2020

Resumen: El presente estudio analiza la situación en la que se encuentran los profesionales en formación del Servicio Murciano de Salud (SMS), ante el estado de emergencia sanitaria decretado por la pandemia del nuevo coronavirus 2019-nCoV. Para ello se ha realizado un estudio descriptivo a través de la recogida de datos mediante formulario directo, canalizado a través de la red del Comité de Empresa, habiéndose obtenido respuesta de al menos un residente de cada uno de los Servicios que conforman el SMS y alcanzando un tamaño de muestra que permite la total representatividad del colectivo a estudio. Se trata de un estudio que destaca por la rapidez de reclutamiento de la muestra y la rapidez en su redacción, lo que sirve como una importante herramienta para condicionar futuras políticas y decisiones en el ámbito de los profesionales en formación. La falta de acceso a material de protección por más de la mitad de la población a estudio junto con dificultades para el acceso a información actualizada por parte de la administración, son algunos de los resultados obtenidos tras el análisis de las respuestas. En general, existe una mayoría de residentes que continúan con su labor asistencial, que mantienen el número de guardias mensuales, que piensa que recibe formación oficial adecuada y que se está realizando un plan de contingencia apropiado.

Palabras clave: Formación Sanitaria Especializada; MIR; COVID-19; Estrés; Plan de Contingencia; Guardias

\begin{abstract}
The present study intends to analyze the situation of professionals in training in the Murcian Health Service (SMS) during the state of sanitary emergency in Spain due to the pandemic of the new coronavirus 2019-nCoV. To achieve this objective, a descriptive study has been carried out through the collection of data via
\end{abstract}


direct form, channeled through the network of the Comité de Empresa. A response from at least one resident of each of the Services of the SMS has been obtained. This has allowed a sample size that represents a large amount of the group under study. This is a study that stands out for the rapid acquisition and analysis of the sample, which serves as an important tool to condition future policies and decisions in the field of professionals in training. The lack of access to protection material for more than half of the population under study, as well as difficulties in accessing updated information from the administration are some of the results obtained after analyzing the responses. Overall, there is a majority of residents that continue with their work assistance and which maintain the same number of 24-hour shifts. It is also a main opinion that there is an appropriate health training and contingency plan against COVID-19.

Keywords: Medical Specialized Training, MIR, COVID-19, Stress; Contingency Plan; 24 hour service

\section{Introducción}

El presente estudio pretende analizar, ante el estado de emergencia sanitaria española (BOE Orden SND/232/2020) decretado por la pandemia del nuevo coronavirus 2019-nCoV (1), la situación laboral de los profesionales en formación del Servicio Murciano de Salud (SMS).

Se trata de un informe que se aprovecha de la capacidad que se dispone desde las instituciones que representan a los profesionales sanitarios en formación de la Región de Murcia (Ilustre Colegio Oficial de Médicos de Murcia y Comité de Empresa del Servicio Murciano de Salud) para coordinar y acceder en tiempo real a la información referente del colectivo y comprobar de esa manera la situación actual que vive dicho grupo en la aplicación de las diferentes medidas que le afectan. La capacidad para implicar al mencionado colectivo y hacer que sea consciente de su rol dentro del sistema es tal, que se trata de una herramienta poderosísima en situaciones de crisis como la que estamos viviendo por la pandemia mundial por coronavirus (2019-nCoV).

La formación Sanitaria Especializada (FSE) que acredita y titula a los profesionales sanitarios para la realización de las diferentes especialidades sanitarias, ya sean las médicas, enfermeras, etcétera, es llevada a cabo en España por una red hospitalaria, de Atención Primaria y Servicios Centrales con una coordinación central del Ministerio de Sanidad (2). Este ministerio es el que se encarga de acreditar las diferentes unidades docentes y sus programas formativos para habilitar la respectiva formación de residentes de los diferentes programas: Médico Interno residente (MIR), Enfermero Interno Residente (EIR), Farmacéutico Interno residente (FIR), Psicólogo Interno residente (PIR), Químico Interno Residente (QIR), Biólogo Interno Residente (BIR) y Radiofísico Interno Residente (RFIR) (3).

La información que aquí recogemos presenta pues una "radiografía" esencial de cómo se ha establecido la organización de los servicios en los que se forman los residentes y cómo ésta ha sido percibida por ellos; además, se ha analizado su utilización como parte de los recursos humanos de aquéllos. Se establece así un análisis entre todas las especialidades que poseen residentes en formación y con representación de la totalidad de las áreas de salud que conforman la Región de Murcia. En este sentido, durante el transcurso de esta crisis sanitaria, han sido numerosas las recomendaciones oficiales de actuación (4-5) y de protección de los trabajadores frente al desarrollo de sus labores dentro de los servicios sanitarios (4-6). 
El objetivo principal es estudiar los aspectos relacionados con la formación recibida, el material de autoprotección para evitar el contagio y la situación de estrés percibida ante la infección por el nuevo coronavirus COVID-19. El objetivo secundario es analizar la carga de trabajo medida por el número de guardias promedio y valorar su diferencia con respecto a la situación preemergencia.

\section{Métodos.}

Para la obtención de estos datos se creó un formulario a través de la plataforma abierta Google Drive ${ }^{\circledR}$ con los ítems que se consideraron relevantes y se distribuyó entre los residentes de diferentes servicios y perfiles sanitarios de las diferentes áreas de salud de la Región de Murcia. Teniendo en cuenta una población diana estimada de aproximadamente 1.000 residentes y habiendo recibido 264 respuestas y asumiendo un intervalo de confianza (IC) de 95\%, con un margen de error del 5\% y una heterogeneidad del $65 \%$, la muestra necesaria sería de 261 participantes, resultando así el estudio estadísticamente significativo.

El periodo de recogida de datos corresponde a los días 21, 22 y 23 de marzo de 2020. Para cada especialidad se recogieron las siguientes variables:

- Área de salud a la que pertenece. Son las siguientes:

- Área I.- Murcia Oeste (Hospital Clínico Universitario Virgen de la Arrixaca). Área II.- Cartagena (Complejo Hospitalario Universitario de Cartagena).

Área III.- Lorca (Hospital Rafael Méndez).

Área IV.- Noroeste (Hospital Comarcal del Noroeste).

Área V.- Altiplano (Hospital Virgen del Castillo).

Área VI.- Vega Media del Segura (Hospital General Universitario Morales Meseguer).

- Área VII.- Murcia Este (Hospital General Universitario Reina Sofía).

- Área VIII.- Mar Menor (Hospital General Universitario de Los Arcos Mar Menor).

- Área IX.- Vega Alta del Segura (Hospital de la Vega Lorenzo Guirao).

- Especialidad sanitaria. La totalidad de las especialidades sanitarias para los diferentes modelos de formación sanitaria (PIR, FIR, BIR, QIR, EIR o MIR) se exponen en la Tabla 1.

- Año de residencia: R1, R2, R3, R4 o R5.

- Realización, por parte del Servicio, de un plan de contingencia que permita realizar una actividad laboral productiva.

- Si ha habido reubicación de los residentes de dicha especialidad.

- Estudio del número de guardias promedio.

- Si se está proporcionando el material de autoprotección para evitar el contagio por COVID-19.

- Si se está proporcionando formación específica suficiente por los canales oficiales para afrontar esta situación.

- Valoración del grado de estrés laboral en el puesto de trabajo actualmente. Utilizándose para ello una escala numérica con valores del 1 al 10.

- Otras aportaciones. Texto libre a rellenar por el encuestado con propuestas de mejora o preocupaciones sobre la situación que está viviendo.

Una vez obtenidos los datos de forma cuantitativa a través de las respuestas voluntarias por parte de los residentes que hubieran recibido la encuesta por diferentes medios (redes sociales, correo electrónico), se realizó un listado con los servicios de los que no se había obtenido información en esa primera fase, realizándose una segunda fase donde se procede al envío de encuestas con el mismo formato, dirigidas hacia residentes de los servicios de los que no se disponía 
información, obteniéndose finalmente un porcentaje del $100 \%$ del total de los servicios en los que participan residentes en el SMS.

En el caso del porcentaje de participación de cada uno de los servicios mencionados previamente, se ha realizado a través de la división de las respuestas obtenidas por el número total de residentes en la Región de Murcia para esa especialidad. Dicho valor se ha aproximado a la siguiente unidad en el caso de que saliera con decimales. El total de residentes de cada una de las especialidades se ha obtenido de la Dirección de Recursos Humanos del SMS, ya que se trató de documentación que tuvo que mandar al Ministerio de Sanidad para la elaboración del plan de recursos humanos ante la situación de emergencia sanitaria determinada.

Se valoran las diferencias de manera global a nivel de la Región de Murcia, en función de las áreas, de los servicios y especialidades en cada una de ellas (MIR, EIR, PIR, FIR, QUIR, BIR, RFIR).

\section{Resultados}

Tras realizar un análisis pormenorizado de las respuestas, se detallan a continuación los resultados agrupados según las preguntas que se formularon.

\subsection{Preguntas y resultados asociados.}

\subsection{1. Área donde desarrolla el profesional en formación su actividad.}

Se recibieron un total de 264 respuestas que se distribuyen por las diferentes áreas del SMS tal y como puede observarse en la Figura 1. El mayor número de respuestas coincide con las áreas que más profesionales en formación tienen, como son el Área I (Murcia Oeste.-Hospital Clínico Universitario Virgen de la Arrixaca) y Área II (Cartagena.-Complejo Hospitalario Santa Lucía-Santa María del Rosell).

\subsubsection{Especialidad en la que se encuentra el personal en formación encuestado.}

En cuanto a las especialidades en las que se encuentran los profesionales en formación encuestados destaca en la Figura 2 que el mayor porcentaje del total $(32,8 \%)$ corresponde a residentes de la especialidad médica de Medicina Familiar y Comunitaria. Así mismo es reseñable cómo especialidades de enfermería se encuentran también representadas en esta muestra, si bien con respecto al porcentaje total de los encuestados es menor, correspondiendo por ejemplo para los EIR de Enfermería Familiar y Comunitaria un 8,2\% del total.

En relación al porcentaje total de especialistas en formación que se dan en las diferentes especialidades, y teniendo en cuenta un documento aportado por la Dirección de Recursos Humanos del SMS que cifra en 1.023 la totalidad de residentes de la Región, el porcentaje de respuestas con respecto al total de residentes de los diferentes servicios varía en el rango del $10 \%$ al $100 \%$ y viene reflejado en las Tablas $1 \mathrm{y} 2$.

\subsubsection{Año de residencia en el que se encuentra el profesional en formación encuestado.}

En relación al año de residencia en el que se encuentran los encuestados, se puede observar en la Figura 3 cierto equilibrio entre los dos primeros años (R1 y R2) siendo el 30,3\% y 33\% de la muestra, respectivamente. Este porcentaje disminuye progresivamente al encontrarse un menor número de residentes en los sucesivos años de especialidad, correspondiendo a R3 un 22,1\%, R4 el 11,2\% y R5 que desciende hasta el 3,4\% de la muestra. 
3.1.4. Medidas de reubicación adoptadas por los servicios para adaptar la actividad del profesional en formación.

La Figura 4 muestra que el $83 \%$ de los residentes encuestados considera que su servicio ha llevado a cabo un plan de contingencia adecuado frente a la situación actual y la posible futura reorganización de profesionales. El mayor número de respuestas se da en el Área I, en la que el 15,9\% considera inadecuado o inexistente el plan de contingencia, hecho que se puede observar en la Figura 4.1. El resultado diferenciado del resto de áreas se recoge en la Tabla 3.

En relación a las medidas adoptadas para reubicar a los residentes de la Región de Murcia analizadas en la Figura 5, se observa que 117 residentes sobre el total de los 264 encuestados realiza su labor asistencial por turnos y en diferentes equipos.

Por otro lado, un total de 110 residentes sobre los 264 trabaja diariamente rotando por distintos puestos según las necesidades de su servicio, 8 residentes del total de encuestados han sido reclamados por otros servicios y 49 residentes siguen una distribución diferente a las opciones de nuestro cuestionario.

3.1.5. Adaptación de la carga laboral en número de guardias tras el decreto de emergencia sanitaria.

La mayoría de los residentes encuestados mantiene el mismo número de guardias, 201 de los 264 encuestados, desde la declaración del estado de alarma por COVID-19 (Figura 6). El 26\% del total han visto modificada su jornada complementaria; el 30\% la han visto incrementada por encima de 5 guardias mensuales y el $70 \%$ reducida.

\subsubsection{Acceso a material de protección por parte de los profesionales en formación frente a COVID-19 según recomendaciones oficiales.}

El 50,9\% de los residentes indican no tener acceso a material de protección adecuado frente al COVID-19 (Figura 7).

3.1.7. Percepción de la disponibilidad de recursos formativos en relación al COVID-19 a través de canales oficiales en los profesionales en formación del SMS.

Más de la mitad de los residentes considera que ha habido una difusión y proporción de la información adecuada al momento. Destaca en la Figura 8 que el $39,3 \%$ considere que no ha sido formado, mediante los canales oficiales, en lo necesario ante esta pandemia.

3.1.8. Percepción actual del nivel de estrés en el desarrollo de la actividad profesional de los residentes de la Región de Murcia.

Basándonos en una escala de percepción subjetiva del estrés del 1 al 10, destaca una autovaloración mayoritaria en niveles de 7 y 8 tal y como muestra la Figura 9 . De los 264 residentes encuestados, 62 consideran tener un nivel de estrés con puntuaciones entre 9 y 10. Por otro lado, del total de residentes más de la mitad de los mismos expone percibir un nivel de estrés superior a los niveles 5 y 6 , siendo minoritario el porcentaje de aquellos que reflejan un nivel de estrés por debajo del valor 4 sobre 10 . 


\subsection{Figuras.}

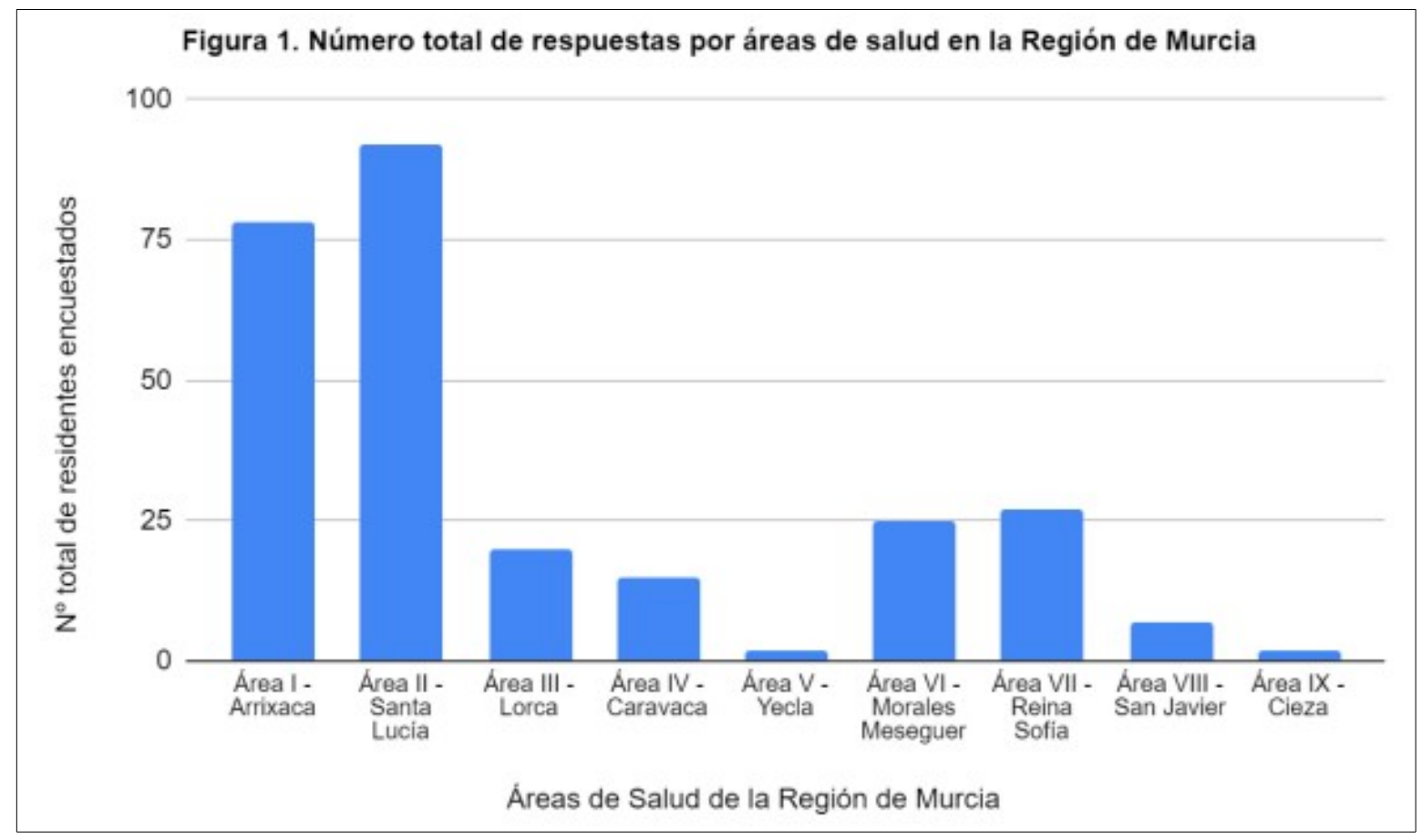

Figura 2. Porcentaje de las respuestas obtenidas de las distintas especialidades de la Región de Murcia*

"Existen otras muchas especialidades presentes en el grálico con sectores más pequeños las cuales no aparecen señaladas con texto y su correspondiante porcentaje

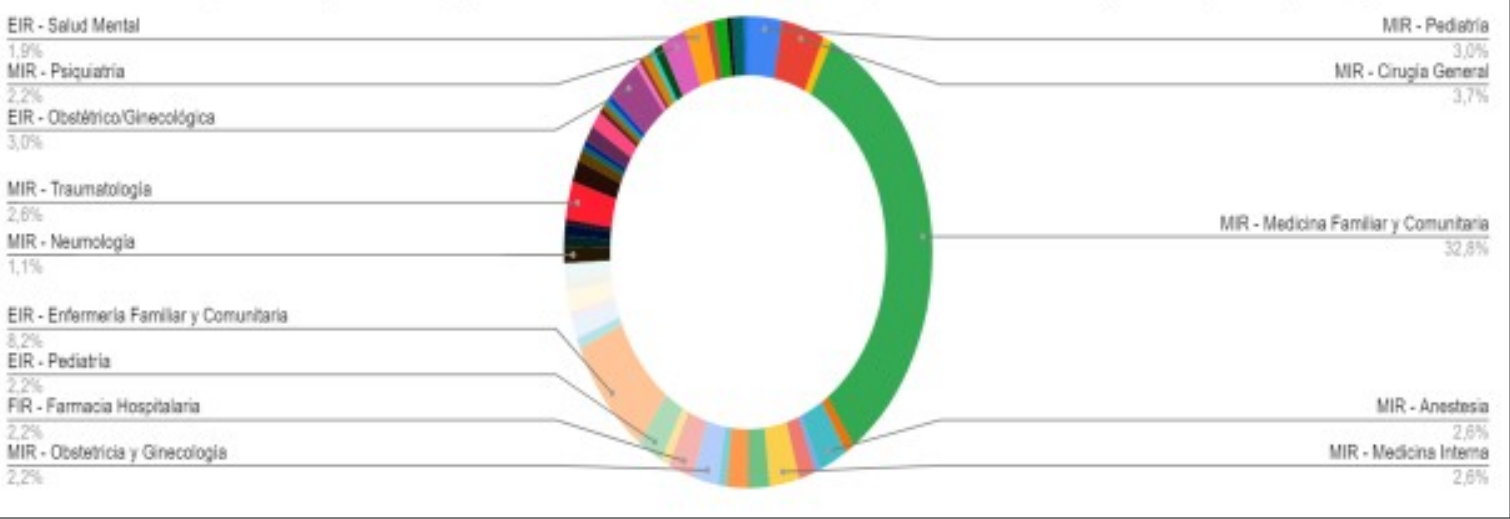

Figura 3. Porcentaje de los encuestados según el año de especialidad

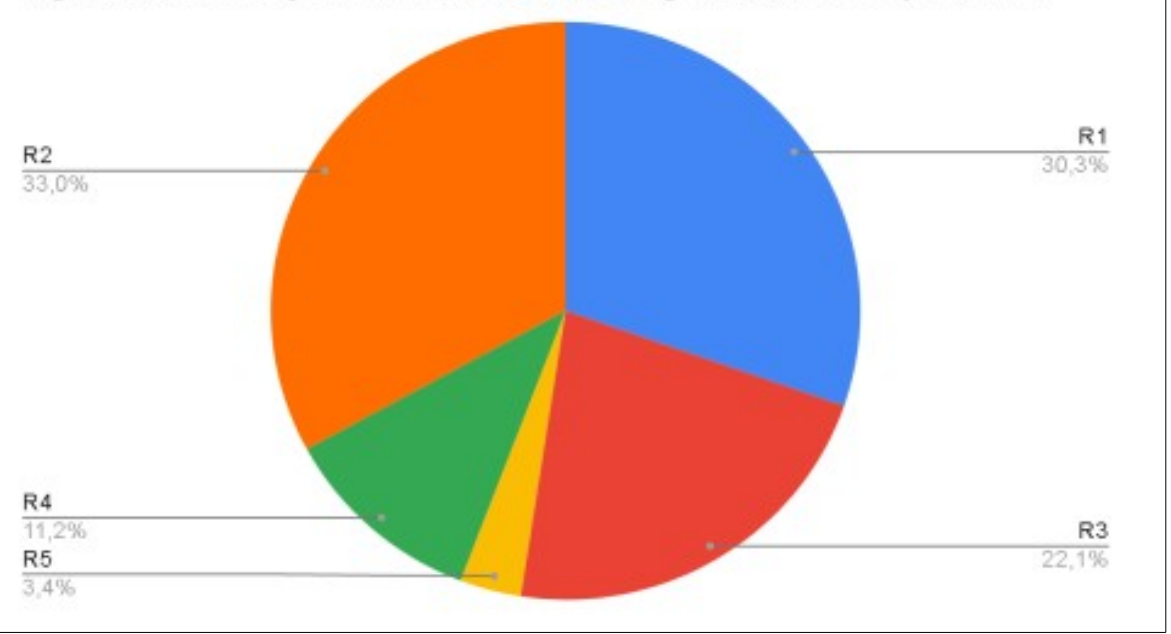


Figura 4. ¿Ha realizado tu servicio un plan de contingencia que te permita realizar una actividad laboral productiva?

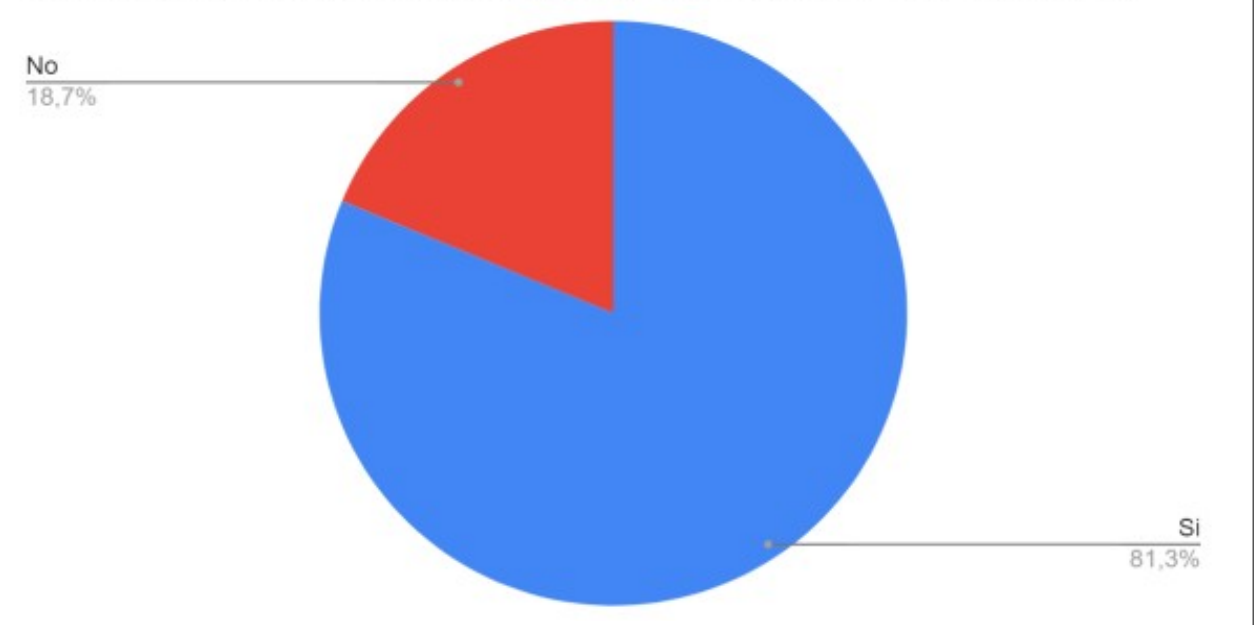

Figura 5. Diferencias en las medidas adoptadas para reubicar a los residentes de la Región de Murcia

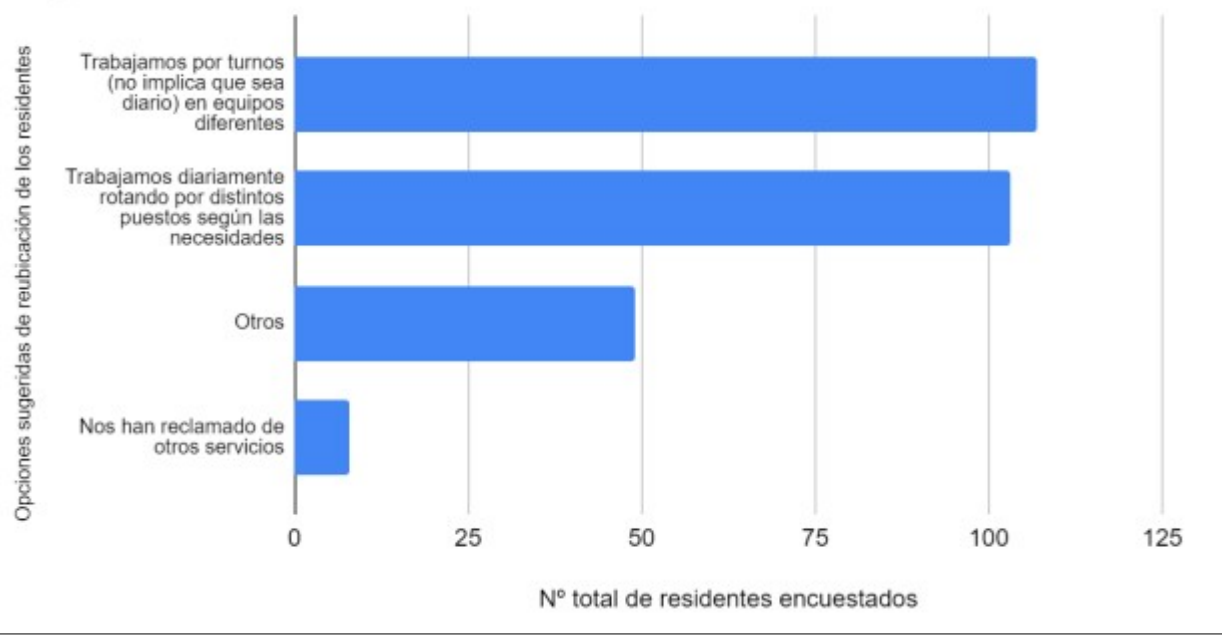

Figura 6. Variaciones en el número de guardias realizadas por los residentes de la Región de Murcia

250

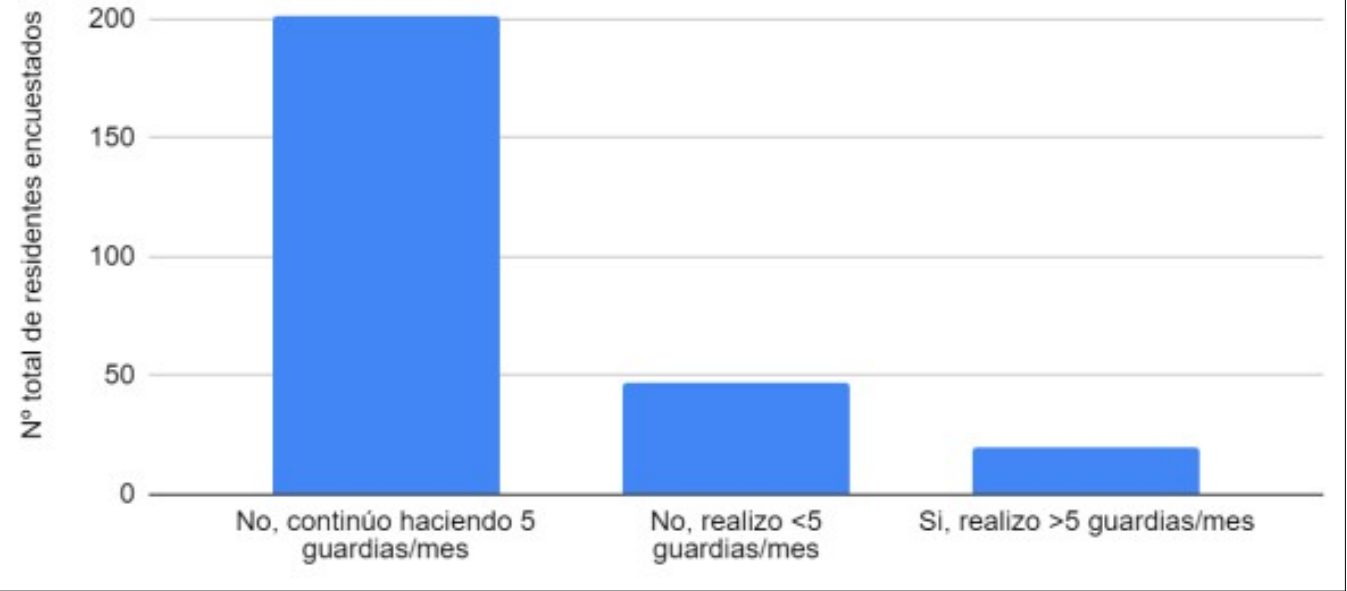



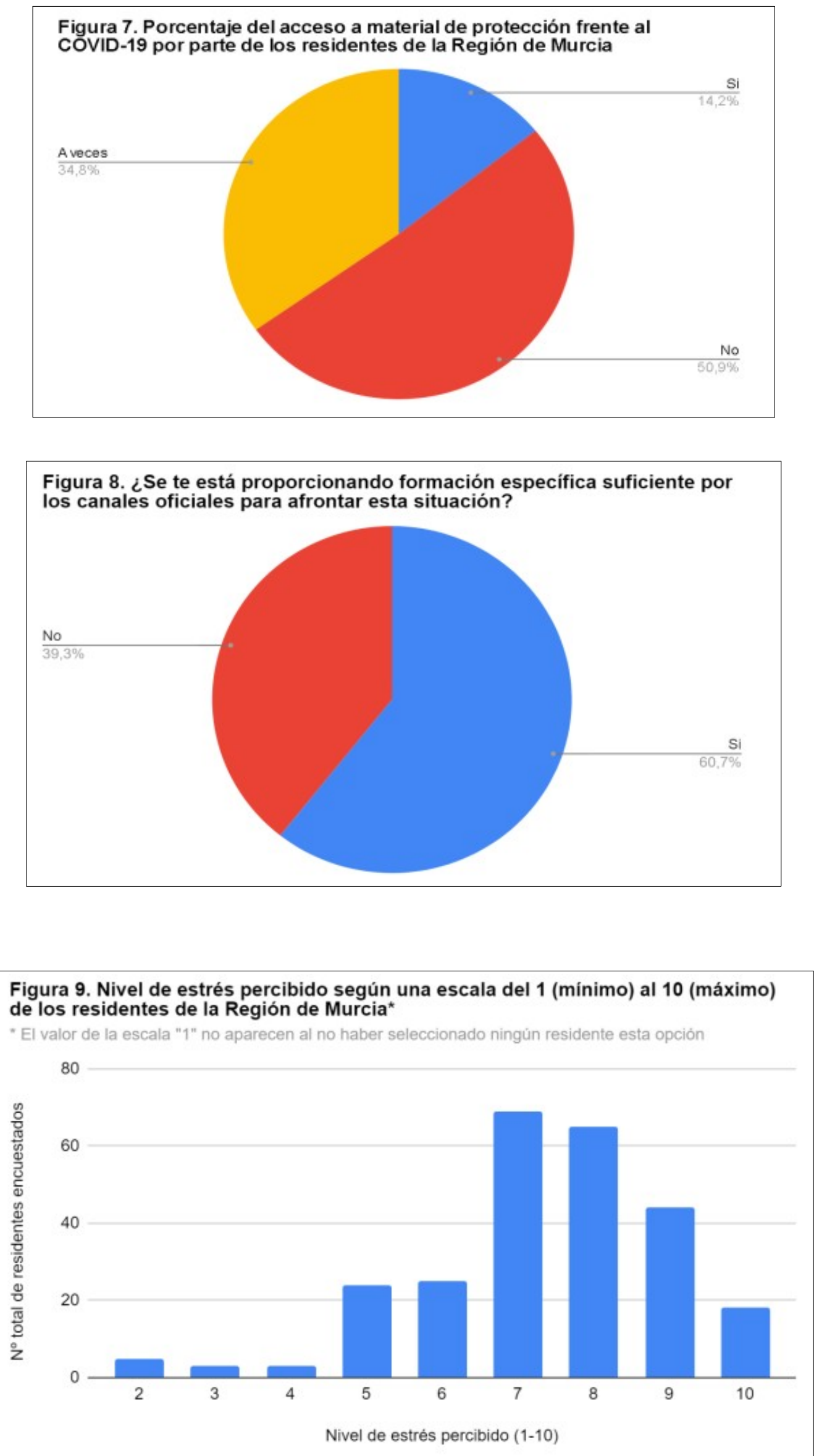


\subsection{Tablas.}

Tabla 1. Porcentaje de respuestas obtenidas por las especialidades médicas (MIR) entre el total de residentes que posee cada una de las especialidades.

\begin{tabular}{|c|c|c|}
\hline Alergología (34\%) & $\begin{array}{c}\text { Anestesia y Reanimación } \\
(15 \%)\end{array}$ & Aparato Digestivo (23\%) \\
\hline Cardiología (20\%) & Cirugía Cardiovascular (34\%) & $\begin{array}{c}\text { Cirugía General y del Ap. } \\
\text { Digestivo (34\%) }\end{array}$ \\
\hline $\begin{array}{c}\text { Cirugía Oral y Maxilofacial } \\
\text { (40\%) }\end{array}$ & $\begin{array}{c}\text { Cirugía Ortopédica y } \\
\text { Traumatología (23\%) }\end{array}$ & Cirugía Pediátrica (34\%) \\
\hline $\begin{array}{c}\text { Cirugía Plástica (20\%) } \\
\text { Hematología (25\%) }\end{array}$ & $\begin{array}{c}\text { Medicina Física y } \\
\text { Rehabilitación (13\%) }\end{array}$ & Medicina del Trabajo (23\%) \\
\hline $\begin{array}{c}\text { Medicina Familiar y } \\
\text { Comunitaria (30\%) }\end{array}$ & Medicina Intensiva (15\%) & Medicina Interna (21\%) \\
\hline Medicina Preventiva y Salud \\
Pública (10\%)
\end{tabular}

Tabla 2. Porcentaje de respuestas obtenidas por especialidades no médicas entre el total de residentes que posee cada una de las especialidades.

\begin{tabular}{|c|c|c|}
\hline $\begin{array}{c}\text { EIR-Enfermería del Trabajo } \\
(50 \%)\end{array}$ & $\begin{array}{c}\text { EIR-Enfermería Familiar y } \\
\text { Comunitaria (52\%) }\end{array}$ & $\begin{array}{c}\text { EIR-Enfermería Pediátrica } \\
(55 \%)\end{array}$ \\
\hline $\begin{array}{c}\text { EIR-Enfermería Salud Mental } \\
(50 \%)\end{array}$ & $\begin{array}{c}\text { EIR-Enfermería Obstétrico } \\
\text { - Ginecológica (30\%) }\end{array}$ & FIR-Análisis Clínicos (20\%) \\
\hline $\begin{array}{c}\text { FIR-Farmacia Hospitalaria } \\
(25 \%)\end{array}$ & FIR-Inmunología (25\%) & $\begin{array}{c}\text { FIR-Microbiología y } \\
\text { Parasitología (34\%) }\end{array}$ \\
\hline BIR-Análisis Clínicos (25\%) & PIR-Psicología Clínica (21\%) & $\begin{array}{c}\text { QIR-Bioquímica Clínica } \\
(100 \%)\end{array}$ \\
\hline
\end{tabular}


Tabla 3. Porcentaje de medidas de reubicación adoptadas en las distintas áreas de Salud.

\begin{tabular}{|c|c|}
\hline Area I & $84,1 \%$ \\
\hline Area II & $80 \%$ \\
\hline Area III & $75 \%$ \\
\hline Area IV & $100 \%$ \\
\hline Area V & $100 \%$ \\
\hline Area VI & $83,3 \%$ \\
\hline Area VII & $85,7 \%$ \\
\hline Area VIII & $50 \%$ \\
\hline Area IX & $100 \%$ \\
\hline
\end{tabular}

\section{Discusión}

El objetivo de este trabajo fue evidenciar en un tiempo inmediato, tras el decreto de Emergencia Sanitaria emitido por el Gobierno de España (1), y durante el transcurso de la subsecuente crisis sanitaria, la situación de los profesionales en formación del Servicio Murciano de Salud en cuanto a la capacidad de acceso a formación sobre el COVID-19 y a material de protección individual o el conocimiento de los planes de contingencia propuestos por las diferentes gerencias de área (7). Todo ello para servir de base en la toma de decisiones y actuaciones que se debieran realizar por parte de las administraciones competentes.

La representatividad de la muestra alcanzada permite extrapolar los resultados al total de los residentes actualmente trabajando para el Servicio Murciano de Salud. De los 264 resultados, la mayor parte se concentra en las dos áreas con mayor número de residentes, las áreas I y II. De las respuestas recibidas, al menos un tercio pertenecen a residentes de Medicina Familiar y Comunitaria, siendo esta especialidad junto con Pediatría y Enfermería Familiar y Comunitaria, un dique fundamental en atención primaria que realiza el seguimiento domiciliario de más de 30.000 casos sospechosos de COVID-19 (8).

En el caso de la FSE, son numerosos los agentes que intervienen en la misma, yendo desde las propias unidades docentes hospitalarias y extrahospitalarias y sus respectivos servicios clínicos hasta las propias gerencias de áreas. Muchas veces, la información y las órdenes se van sucediendo de forma simultánea entre las diferentes estructuras, entrando en muchas ocasiones en colisión unas con otras. Es por ello importante mencionar cómo la coordinación en las situaciones de emergencia sanitaria entre los diferentes niveles jerárquicos dentro de un sistema de salud (5-6) es clave para que el conocimiento de las medidas a adoptar lleguen de forma clara y concisa a los profesionales sobre las que deben ejecutarse. A nivel de formación, más de la mitad de los residentes considera que ha habido una difusión y proporción de la información adecuada al momento, no obstante mermada por la confusión que generan mensajes contradictorios en cuanto a actuación y protocolos en constante modificación. Es reseñable que el 39,3\% refiere que no ha sido formado mediante los canales oficiales en lo necesario ante esta pandemia.

Encontramos que una amplia mayoría, tanto a nivel hospitalario como de atención primaria, considera adecuado el plan de contingencia realizado por su servicio. Es 
preciso tener en cuenta a la hora de interpretar estas gráficas que la pregunta formulada al respecto puede dar lugar a respuestas subjetivas y se valora no sólo la existencia o no del mencionado plan sino la capacidad de llegar a los profesionales del servicio, en este caso los residentes. El bajo número de respuestas en ciertas áreas dota de mayor peso al factor subjetivo, encontrando diferencias marcadas con respecto a la media (nótese en las áreas IV, V y VIII).

En relación a las medidas adoptadas para reubicar a los residentes de la Región de Murcia, se observa que 117 sobre el total de encuestados, realiza su labor asistencial por turnos y en diferentes equipos siguiendo una fórmula que permite reducir contacto entre profesionales y de la misma forma sustituir al equipo por completo en caso de enfermedad de uno de los miembros. Por otro lado, un total de 110 residentes sobre los 264, trabaja diariamente rotando por distintos puestos según las necesidades de su servicio. El número de guardias mensuales que realiza cada residente, si bien el formulario fue realizado al inicio de la situación, se mantiene igual en un $74 \%$ de los encuestados. En el $26 \%$ que han sufrido cambios, en su mayoría han ido a favor de reducir el número de guardias, siendo esto un claro detrimento al ser las guardias médicas en algunos casos hasta el 50\% de la retribución mensual del residente.

El Ministerio de Sanidad, según la Guía de Actuación frente a COVID-19 en los profesionales sanitarios y socio-sanitarios (9-10), considera que todo personal sanitario que atienda una sospecha o confirmación de Covid-19 debe llevar un equipo de protección individual compuesto de protección ocular, bata, guantes y mascarilla (quirúrgica o, en caso de procedimientos que generen aerosoles, FFP2 o FFP3). Al menos la mitad de los residentes encuestados no cuentan con material adecuado en su labor asistencial. Es evidente el escaso acceso a material adecuado, tanto en cuestiones de equipos completos de protección individual como en componentes independientes, ya sean mascarillas, guantes, gafas y soluciones hidroalcohólicas, entre otros. Esto se ve reflejado en la mayoría de las especialidades y no siendo el caso de un servicio aislado.

La alta carga de trabajo así como la ausencia de protección adecuada son algunas de las causas que incrementan el ya presente burnout entre personal sanitario. Numerosos expertos advierten de la extrema necesidad de prevenir y tratar el trauma emocional que supone esta crisis sanitaria (11). Una amplia mayoría de encuestados refiere un nivel de estrés de 7 o más sobre 10. Destaca además que un $20 \%$ se encuentra en un nivel cercano al límite superior. Una de las limitaciones de este estudio es no haber usado cuestionarios validados de burnout entre el profesional sanitario, tales como el Maslach Burnout Inventory, siendo necesarios estudios futuros que pongan de manifiesto las repercusiones emocionales derivadas de esta situación excepcional.

\section{Conclusiones}

- Existe una percepción mayoritaria por parte de los residentes, tanto a nivel general como por áreas de salud, de que sus servicios han elaborado un plan de contingencia frente a la actual crisis por COVID 19, si bien existen diferencias entre las distintas áreas de salud.

- Más del 50\% de los residentes continúan con su labor asistencial, a diario o por turnos, adaptándose a las necesidades que requieran. Otros quedan a disposición de su servicio para incorporarse a la actividad laboral según evolucione la situación sanitaria vigente.

- $\quad$ El 75\% de los residentes encuestados de la Región continúan haciendo 5 guardias mensuales. Sin embargo, encontramos un porcentaje de residentes que deberá 
realizar más de 5 guardias por necesidades del servicio y en los que es preciso asegurar que en este periodo extraordinario se produzca la remuneración de las mismas.

- Sólo el 14,2\% de los sanitarios en formación consideran que cuentan con el material suficiente para evitar contagios. Expresando el 50,9\% de los mismos directamente no tener acceso a dicho material mientras que el 39,3\% comenta únicamente disponer de ellos a veces.

- El 60\% considera que está recibiendo formación adecuada de cara a afrontar la pandemia por COVID-19.

- Según los resultados obtenidos, los residentes de la Región de Murcia presentan niveles altos de estrés por la situación actual de pandemia por COVID-19. En una escala del 1 al 10, la sensación percibida de estrés laboral actualmente supera el 7,5 en más de la mitad de los encuestados, no siendo despreciable el número de residentes que se sienten con un nivel de estrés de 9 ó 10 sobre una escala máxima de puntuación 10 .

Material suplementario: no hay.

Financiación: No ha habido financiación.

Agradecimientos: Se expresa un especial agradecimiento a todos los profesionales sanitarios y en particular, a aquellos que se encontraban en formación durante el estado de emergencia sanitaria, ya que con su esfuerzo e implicación máxima han conseguido ofrecer la mejor asistencia sanitaria en una época difícil. Una especial mención al grupo de trabajo de áreas de la Vocalía de Médicos Jóvenes y Promoción de Empleo del Ilustre Colegio Oficial de médicos de la Región de Murcia, que teniendo una visión colectiva hacia la mejora del colectivo en formación, han trabajado por defenderla en esta situación excepcional.

Declaración de conflicto de interés: Los autores declaran no tener ningún conflicto de interés.

\section{Referencias}

1. Boletín Oficial del Estado. Orden SND/232/2020, de 15 de marzo, por la que se adoptan medidas en materia de recursos humanos y medios para la gestión de la situación de crisis sanitaria ocasionada por el COVID-19. https://www.boe.es/boe/dias/2020/03/15/ index.php?e $=4335$

2. Página web del Ministerio de Sanidad. Disponible online: https://fse.mscbs.gob.es/fseweb/ view/index.xhtml (visitado el 06/04/2020).

3. Boletín Oficial del Estado. Orden SCB/925/2019, de 30 de agosto, por la que se aprueba la oferta de plazas y la convocatoria de pruebas selectivas 2019 para el acceso en el año 2020, a plazas de formación sanitaria especializada para las titulaciones universitarias de grado/licenciatura/diplomatura de Medicina, Farmacia, Enfermería y ámbito de la Psicología, la Química, la Biología y la Física. https://www.boe.es/diario boe/txt.php? $\underline{i d}=$ BOE-A-2019-12897

4. Cherrie JW, Levy L. Managing Occupational Exposure to Welding Fume: New Evidence Suggests a More Precautionary Approach is Needed. Annals of Work Exposures and Health 2020; 64: 1-4. https://doi.org/10.1093/annweh/wxz079

5. Ministerio de Sanidad. Manejo en atención primaria del COVID-19. Versión del 17 de marzo de 2020. https://www.mscbs.gob.es/profesionales/saludPublica/ccayes/ alertasActual/nCov-China/documentos/Manejo primaria.pdf

6. Ministerio de Sanidad. Procedimiento de actuación para los servicios de prevención de riesgos laborales frente a la exposición al nuevo coronavirus (SARS-COV-2). Versión del 8 de marzo de 2020. https://www.mscbs.gob.es/profesionales/saludPublica/ ccayes/alertasActual/nCov-China/documentos/PrevencionRRLL_COVID-19.pdf

7. Ministerio de Sanidad. Informe sobre los aspectos éticos en situaciones de pandemia: El SARS-CoV-2. 2 de abril de 2020. https://www.mscbs.gob.es/profesionales/saludPublica/ ccayes/alertasActual/nCov-China/documentos/200403-INFORME_PANDEMIA-FINALMAQUETADO.pdf 
8. Consejería de Salud, Región de Murcia. Disponible online: https://www.murciasalud.es/noticias.php?op=ver\&id=459132\&idsec $=66 \quad$ (visitado el 07/04/2020).

9. Ministerio de Sanidad. Guía de Actuación frente a COVID-19 en los profesionales sanitarios y sociosanitarios. Documento técnico. Versión 31 de marzo de 2020. https://www.mscbs.gob.es/profesionales/saludPublica/ccayes/alertasActual/nCov-China/ documentos/Protocolo_Personal sanitario COVID-19.pdf

10. World Health Organization. Novel Coronavirus (2019-nCoV) technical guidance. Disponible online en: https://www.who.int/emergencies/diseases/novel-coronavirus2019/technical-guidance (visitado el 07/04/2020).

11. Li Z, Ge J, Yang M, Feng J, Qiao M, Jiang R, Bi J, Zhan G, Xu X, Wang LZ. Vicarious traumatization in the general public, members, and non-members of medical teams aiding in COVID-19 control. Brain, Behavior and Immunity 2020; in press. https://doi.org/10.1016/j.bbi.2020.03.007

(C) 2020 por los autores. Enviado para su posible publicación en acceso abierto bajo los términos y condiciones de la licencia Creative Commons Attribution (CC BY) (http://creativecommons.org/licenses/by/4.0/). 